\title{
Haemodynamic patterns in patients with scorpion envenomation
}

\author{
D R Karnad
}

\begin{abstract}
Objective-To study cardiovascular haemodynamics following scorpion envenomation.

Setting-Intensive care unit of a university hospital.

Patients-Eight patients with Indian red scorpion (Mesobuthus tamulus) stings.

Intervention-Captopril (6.25 to $12.5 \mathrm{mg}$ orally) every 30 minutes until pulmonary oedema resolved.
\end{abstract}

Main outcome measures-Haemodynamic data obtained by pulmonary artery catheterisation.

Results-Two haemodynamic patterns were seen. There was a predominant vascular effect in one patient, with severe hypertension, tachycardia, increased systemic vascular resistance index $\left(\right.$ SVRI = 5893 dyn.s. $\left.\mathrm{cm}^{-5}\right)$, and normal cardiac index $\left(2.731 / \mathrm{m}^{2}\right)$. A predominant myocardial effect with left ventricular dysfunction and normal right ventricular function was seen in the other seven patients, with tachycardia, pulmonary oedema, mild hypotension, reduced stroke volume (mean (SD), $25.9(8.3) \mathrm{ml} / \mathrm{m}^{2}$ ), normal SVRI (1812 (831) dyn.s.cm ${ }^{-5}$ ), and increased pulmonary artery wedge pressure $(P A W P=25(4.4) \mathrm{mm} \mathrm{Hg})$. Following mild dehydration pulmonary oedema subsided (PAWP $=14(8.5) \mathrm{mm} \mathrm{Hg}$ ) in three of these patients, but hypovolaemic shock developed (right atrial pressure $(\operatorname{RAP})=1.3 \quad(2.1) \mathrm{mm}$ Hg); pulmonary oedema recurred with rehydration. One patient developed fatal cardiogenic shock with raised PAWP (27 $\mathrm{mm} \mathrm{Hg})$ and RAP (11 mm Hg), and vasodilatation $\left(\right.$ SVRI $=1129$ dyn.s. $\left.\mathrm{cm}^{-5}\right)$. Stroke volume (30.5 (8.7) $\mathrm{ml} / \mathrm{m}^{2}$ ) and cardiac output (4.3 (1.5) $1 / \mathrm{m}^{2}$ ) improved with resolution of pulmonary oedema $\quad(P A W P=14.4$ (4.2) $\mathrm{mm} \mathrm{Hg}$ ) following afterload reduction with captopril.

Conclusions-Mild envenomation causes severe vasoconstriction and hypertension. Severe envenomation produces predominant left ventricular dysfunction with normal systemic vascular resistance manifesting as pulmonary oedema or severe hypotension depending on the fluid balance. Shock due to biventricular dysfunction and vasodilatation occurs terminally.

(Heart 1998;79:485-489)

Keywords: scorpion sting; myocarditis; pulmonary oedema; animal toxin
Scorpion stings are common in tropical and subtropical regions of the world. In western India most cases of envenomation are following stings from Mesobuthus tamulus (red scorpion), ${ }^{12}$ while Heterometrus bengalensis is common in eastern India. ${ }^{3}$ The Androctonus spp and Leirus spp are common in Northern Africa, Middle East and Israel, Parabuthus spp in South Africa, Tityus spp in South America, and Centruroides spp in North and Central America. ${ }^{4}$

Venoms of all species affect the cardiovascular system and may cause fatal pulmonary oedema and cardiac arrhythmias. ${ }^{5}$ Experimental studies have shown that the venom causes cholinergic as well as adrenergic neurone hyperstimulation by its action on presynaptic cell membranes. ${ }^{35}$ It also has a direct effect on the gating mechanisms of excitable membranes. ${ }^{5}$ As a result, there is massive release of catecholamines from sympathetic nerve endings and from the adrenal medulla. ${ }^{67}$ The elevation of circulating catecholamines and angiotensin results in intense vasoconstriction and cardiac stimulation, ${ }^{8}$ increased myocardial oxygen requirements, ${ }^{5}$ and alterations in myocardial perfusion ${ }^{9}$ and metabolism, with hyperglycaemia and an increase in circulating free fatty acids. ${ }^{10}$ Several of these mechanisms, together with a possible direct effect of the toxin on the myocardium, may be responsible for the myocarditis and focal myocardial necrosis seen patients dying from envenomation. ${ }^{11} 12$

Scorpion envenomation in humans is manifested as hypertension in mild cases and hypotension or pulmonary oedema in severe cases. ${ }^{5}$ As there are very few published studies of haemodynamic changes following scorpion envenomation, treatment of these patients has been essentially empirical or based on observations from animal studies. Methods of treatment include vasodilators, ${ }^{13-15}$ specific antivenom serum, ${ }^{16}{ }^{17}$ glucose-insulin infusion, ${ }^{8}$ and inotropic drugs. ${ }^{18}$ The treatment of patients with hypotension and pulmonary oedema, in particular, is not clear. ${ }^{19}$ We therefore studied the haemodynamic alterations following scorpion envenomation and the effects of treatment.

Methods

We studied eight patients with systemic envenomation following scorpion sting admitted to the medical intensive care unit of the King Edward Memorial Hospital, a tertiary referral centre and teaching hospital in Bombay, India. All patients had haemodynamic monitoring after informed consent had been obtained. An
Accepted for publication 22 January 1997 
Table 1 Clinical and laboratory profile of patients with scorpion envenomation

\begin{tabular}{lll}
\hline Variable & Median & Range \\
\hline Age (years) & 20 & 13 to 50 \\
Sting-admission interval $(\mathrm{h})$ & 9 & 1 to 18 \\
Hypertension & $\mathrm{n}=1$ & - \\
Hypotension & $\mathrm{n}=7$ & - \\
Packed cell volume (\%) & 40 & 32 to 46 \\
Total WBC $(1000 / \mu \mathrm{l})$ & 19.1 & 8.6 to 36.5 \\
$\mathrm{PaO}_{2}(\mathrm{kPa})$ & 5.3 & 3.2 to 7.1 \\
$\mathrm{PacO}_{2}(\mathrm{kPa})$ & 4.1 & 2.7 to 6.2 \\
$\mathrm{Arterial} \mathrm{pH}^{\text {Arterial } \mathrm{HCO}}(\mathrm{mmol} / \mathrm{l})$ & 7.40 & 7.21 to 7.49 \\
Blood urea $\mathrm{N}(\mathrm{mmol} / \mathrm{l})$ & 18.3 & 13.1 to 22.3 \\
Serum creatinine $(\mathrm{mmol} / \mathrm{l})$ & 8.2 & 4.6 to 18.2 \\
Serum sodium $(\mathrm{mmol} / \mathrm{l})$ & 0.1 & 0.09 to 0.17 \\
Serum potassium $(\mathrm{mmol} / \mathrm{l})$ & 136 & 127 to 141 \\
Blood glucose $(\mathrm{mmol} / \mathrm{l})$ & 8.4 & 2.5 to 3.8 \\
\hline
\end{tabular}

$\mathrm{N}$, nitrogen; WBC, white blood count.

arterial line was inserted for measurement of blood gases and arterial pressure. A pulmonary artery catheter was inserted and pressures were measured on admission and periodically thereafter. Cardiac output was measured by thermodilution, and cardiac index, systemic vascular resistance, left ventricular stroke work index, and right ventricular stroke work index were calculated.

No antivenom was given as this is not yet available in India. Oral captopril was used to treat hypertension, while patients with hypotension and pulmonary oedema were given oral captopril and intravenous fluids. The pulmonary artery catheter was removed after $48-72$ hours.

\section{Results}

The mean age of the eight patients (four male, four female) was 25.9 years (range 13 to 50 years). They all had leucocytosis, severe hypoxaemia, and mild metabolic acidosis (table 1). Based on the clinical and haemodynamic features seen on admission and the course thereafter, two haemodynamic patterns were observed.

PREDOMINANT VASCULAR EFFECT

This was observed in only one patient and was characterised by tachycardia and severe systemic vasoconstriction resulting in arterial hypertension with a normal cardiac output (table 2). The pulmonary wedge pressure was normal and right atrial pressure was reduced.
Within one hour of administration of captopril, systemic vascular resistance and arterial pressure decreased and stroke volume and cardiac output increased (table 3 ).

\section{PREDOMINANT MYOCARDIAL EFFECT}

The other seven patients presented with severe tachycardia, mild hypotension, cold clammy extremities, tachypnoea, gallop rhythm, and pulmonary oedema with pink frothy or blood tinged sputum and coarse rales. These patients had left ventricular dysfunction with high pulmonary artery wedge pressures and normal systemic vascular resistance. Right ventricular stroke work index and right atrial pressure were normal and there was a large difference between the pulmonary artery wedge pressure and the right atrial pressure (table 2).

A slight variation of this haemodynamic pattern was observed in three patients who had pulmonary oedema on admission, but subsequently developed mild hypovolaemia after four to eight hours - two as a result of profuse sweating and vomiting and one following intravenous injection of frusemide. Pulmonary oedema improved in all three patients. The pulmonary artery wedge pressure became normal but right atrial pressure fell to very low levels and the difference between the two persisted. Cardiac output and mean arterial pressure fell further and circulatory shock deteriorated (table 2). With infusion of fluids, blood pressure improved but pulmonary oedema worsened, so that the patients returned to their original haemodynamic state.

One patient who had pulmonary oedema and mild hypotension on admission developed cardiogenic shock as a terminal event. After initial improvement in haemodynamics with captopril, this patient had progressive deterioration of pulmonary oedema with severe hypotension on day 3 , characterised by left and right ventricular failure - both right atrial and pulmonary artery wedge pressures were very high and the cardiac index was low. Systemic vascular resistance was also severely reduced (table 3). Intravenous dopamine was added but the blood pressure and cardiac output did not improve. This patient died 36 hours after admission.

Table 2 Haemodynamic patterns observed in patients with scorpion envenomation $(n=8)$

\begin{tabular}{|c|c|c|c|c|c|c|}
\hline Variable & Normal range & $\begin{array}{l}\text { Pattern } 1 \\
(n=1)\end{array}$ & $\begin{array}{l}\text { Pattern } 2 \\
(n=8)\end{array}$ & $\begin{array}{l}\text { Pattern } 3 \\
(n=3)\end{array}$ & $\begin{array}{l}\text { Pattern } 4 \\
(n=1)\end{array}$ & $\begin{array}{l}\text { Pattern } 5 \\
(n=7)\end{array}$ \\
\hline Heart rate (beats/min) & $60-100$ & 120 & $141(23)$ & $141(37)$ & 171 & $134(25)$ \\
\hline MAP $(\mathrm{mm} \mathrm{Hg})$ & $75-105$ & 203 & $69(230$ & $57.5(16.1)$ & 47 & $76.1(19.1)$ \\
\hline $\mathrm{RAP}(\mathrm{mm} \mathrm{Hg})$ & $0-5$ & 2 & $7.6(3.6)$ & $2.1(2.1)$ & 11 & $8.8(2.6)$ \\
\hline MPAP (mm Hg) & $10-20$ & 14 & $27.3(6.0)$ & $18.4(10)$ & 27 & $20.5(4.9)$ \\
\hline PAWP (mm Hg) & $5-12$ & 12 & $25.3(4.4)$ & $14(8.5)$ & 24 & $14.4(1.5)$ \\
\hline $\mathrm{CI}\left(1 / \mathrm{min} / \mathrm{m}^{2}\right)$ & $2.8-3.5$ & 2.73 & $3.52(1.08)$ & $3.23(0.49)$ & 2.5 & $4.3(1.5)$ \\
\hline $\operatorname{SVI}\left(\mathrm{ml} / \mathrm{m}^{2}\right)$ & $33-47$ & 23 & $25.9(8.3)$ & $23.4(3)$ & 15 & $30.5(8.7)$ \\
\hline SVRI $\left(\right.$ dyn.s. $\left.\mathrm{cm}^{-5} \cdot \mathrm{m}^{-2}\right)$ & $1700-2400$ & 5893 & $1812(831)$ & $1355(239)$ & 1129 & $1462(850)$ \\
\hline LVSWI (g.m.m²/beat) & $45-60$ & 59.8 & $18.4(8.0)$ & $13.6(1.3)$ & 4.6 & $24.6(5.7)$ \\
\hline PVRI (dyn.s. $\mathrm{cm}^{-5} \cdot \mathrm{m}^{-2}$ ) & $150-250$ & 58.6 & $87.6(50.2)$ & $107.9(54.1)$ & 94.2 & $138(89)$ \\
\hline RVSWI (g.m.m²/beat) & $7-12$ & 3.7 & $7.6(3.7)$ & $5.0(2.8)$ & 3.2 & $4.8(3.4)$ \\
\hline
\end{tabular}

Pattern 1, systemic hypertension; pattern 2, hypotension with pulmonary oedema; pattern 3, hypovolaemia with hypotension; pattern 4 , cardiogenic shock; pattern 5 , effect of vasodilators plus fluid infusion.

Values are means (SD), n, or range.

CI, cardiac index; LVSWI, left ventricular stroke work index; MAP, mean arterial pressure; MPAP, mean pulmonary artery pressure; PAWP, pulmonary artery wedge pressure; PVRI, pulmonary vascular resistance index; RAP, right atrial pressure; RVSWI, right ventricular stroke work index; SVI, stroke volume index; SVRI, systemic vascular resistance index. 
Table 3 Haemodynamic data before and after oral captopril $(12.5 \mathrm{mg})$ in a patient with severe hypertension following scorpion envenomation

\begin{tabular}{|c|c|c|c|c|}
\hline Variable & Normal values & $\begin{array}{l}\text { On admission } \\
\text { (precaptopril) }\end{array}$ & $\begin{array}{l}1 \mathrm{~h} \text { after } \\
\text { captopril }\end{array}$ & $\begin{array}{l}6 \mathrm{~h} \text { after } \\
\text { captopril }\end{array}$ \\
\hline Heart rate (beats/min) & $60-100$ & 120 & 105 & 100 \\
\hline $\mathrm{MAP}(\mathrm{mm} \mathrm{Hg})$ & $75-105$ & 203 & 111 & 126 \\
\hline $\mathrm{RAP}(\mathrm{mm} \mathrm{Hg})$ & $0-5$ & 2 & 3 & 5 \\
\hline MPAP (mm Hg) & $10-20$ & 14 & 12 & 14 \\
\hline PAWP (mm Hg) & $5-12$ & 12 & 10 & 10 \\
\hline CI $\left(1 / \mathrm{min} / \mathrm{m}^{2}\right)$ & $2.8-3.5$ & 2.73 & 2.91 & 3.92 \\
\hline $\operatorname{SVI}\left(\mathrm{ml} / \mathrm{m}^{2}\right)$ & $33-47$ & 23 & 28 & 39 \\
\hline SVRI (dyn.s. $\left.\mathrm{cm}^{-5} \cdot \mathrm{m}^{-2}\right)$ & $1700-2400$ & 5893 & 2974 & 2483 \\
\hline LVSWI (g.m.m ${ }^{-2} /$ beat) & $45-60$ & 59.8 & 38.2 & 83.9 \\
\hline PVRI (dyn.s.cm ${ }^{-5} \cdot \mathrm{m}^{-2}$ ) & $150-250$ & 58.6 & 54.9 & 81.6 \\
\hline RVSWI (g.m.m $\mathrm{m}^{-2}$ /beat) & $7-12$ & 3.7 & 3.4 & 9.5 \\
\hline
\end{tabular}

CI, cardiac index; LVSWI, left ventricular stroke work index; MAP, mean arterial pressure; MPAP, mean pulmonary artery pressure; PAWP, pulmonary artery wedge pressure; PVRI, pulmonary vascular resistance index; RAP, right atrial pressure; RVSWI, right ventricular stroke work index; SVI, stroke volume index; SVRI, systemic vascular resistance index.

EFFECT OF CAPTOPRIL AND FLUID INFUSION All seven patients with pulmonary oedema were given oral captopril, $6.25 \mathrm{mg}$. If blood pressure did not decrease below $80 \mathrm{~mm} \mathrm{Hg}$ and there was no improvement in the pulmonary oedema and pulmonary artery wedge pressure after 30 minutes, another dose of $6.25 \mathrm{mg}$ was given. This was done repeatedly until the pulmonary artery wedge pressure fell $12.5 \mathrm{mg}$ while one required $25 \mathrm{mg}$. There was a significant reduction in systemic vascular resistance and the cardiac output and increase in left ventricular stroke work index. All patients required simultaneous infusion of intravenous fluids to maintain the right atrial pressure at 5-6 mm Hg. Captopril was continued in the same dose six hourly for four to five days. All patients except one had a progressive rise in blood pressure and cardiac output, while pulmonary artery wedge pressure and heart rate gradually returned to normal. One patient developed progressive cardiogenic shock.

Two of the eight patients died. One died of cardiogenic shock; the other was completely relieved of pulmonary oedema after administration of captopril and was haemodynamically stable when he developed multiple episodes of ventricular tachycardia and ultimately resistant ventricular fibrillation on day 3. below $20 \mathrm{~mm} \mathrm{Hg}$. Six patients required

\section{Discussion}

The haemodynamic effects of scorpion envenomation could be broadly divided into two patterns - a predominantly vascular effect and a predominantly myocardial effect.

\section{PREDOMINANT VASCULAR EFFECT}

Canine studies have shown that the initial effect of scorpion venom is to cause tachycardia and hypertension. ${ }^{20}$ One patient in our study had severe hypertension as a result of tachycardia and intense vasoconstriction, with an increase in systemic vascular resistance to four times normal levels. The cardiac output, pulmonary artery pressure, pulmonary artery wedge pressure, and right atrial pressures were normal. However, left ventricular function, as assessed by left ventricular stroke work index, was supernormal. Hypertension and tachycardia may represent the mildest form of cardiovascular involvement in scorpion envenomation, and in a series of 42 Indian patients with Mesobuthus tamulus envenomation Bawaskar and Bawaskar observed hypertension in $43 \% .{ }^{1}$ This has also been described in 33\% of Brazilian patients ${ }^{21}$ and $15 \%$ of Saudi Arabian patients. ${ }^{16}$ These patients respond well to treatment and mortality is extremely low. The various antihypertensive agents tried include prazosin, ${ }^{14}$ nifedepine, and hydrallazine. ${ }^{13} \mathrm{We}$ found captopril to be equally effective and the haemodynamic improvement occurred within one hour of oral administration.

\section{PREDOMINANT MYOCARDIAL EFFECT}

Predominant myocardial dysfunction resulting in acute left ventricular failure was seen in seven patients. While severe vasoconstriction was observed in the predominantly vascular effect, the systemic vascular resistance in patients with myocardial dysfunction was normal. The right ventricle was relatively less affected and there was a marked difference between the left and right ventricular diastolic pressures. These patients could manifest clinically one of two syndromes, depending on the state of hydration.

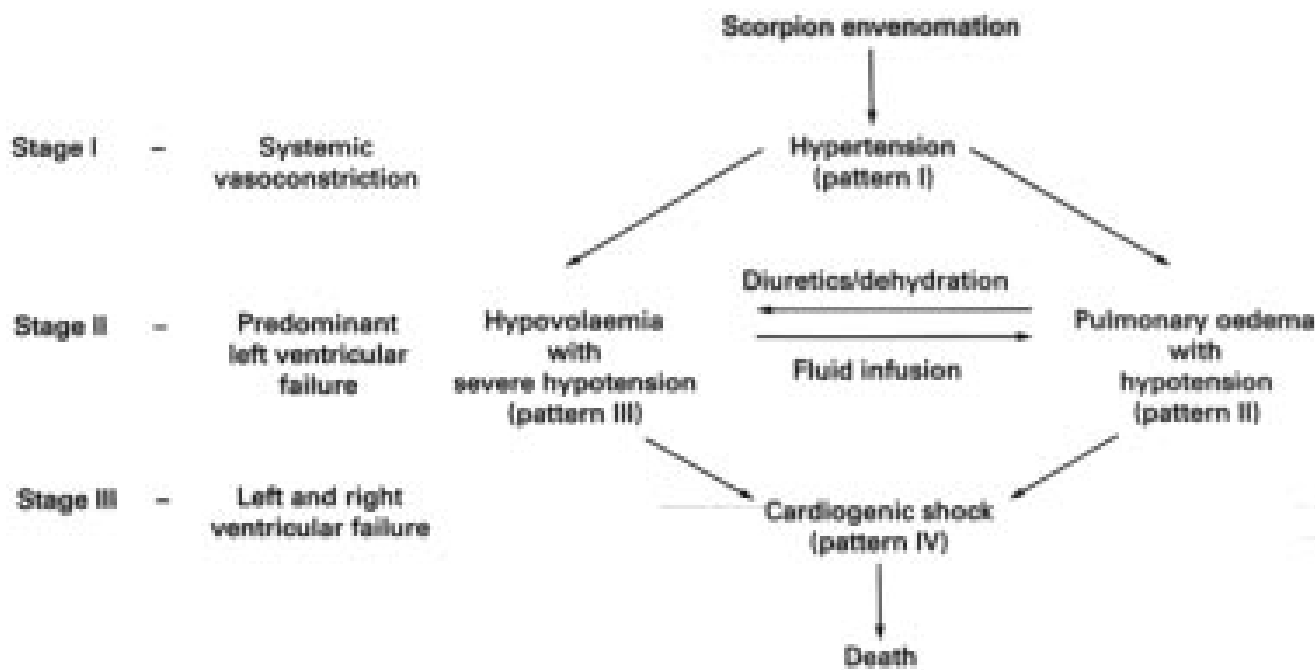

Figure 1 Postulated sequence of events following scorpion envenomation. Haemodynamic changes may result from either predominantly vascular or predominantly myocardial effects of the venom. While a transition from the vascular to the myocardial pattern has been reported in animals, this has not been demonstrated clearly in humans. 
Seven patients with good hydration had mild hypotension but severe pulmonary oedema. Haemodynamically, these patients had raised pulmonary artery wedge pressure and reduced left ventricular stroke work index. The right atrial pressure and right ventricular stroke work index, however, remained normal. Three patients with severe pulmonary oedema on admission subsequently developed mild hypovolaemia - two spontaneously and one as a result of diuretic treatment. The pulmonary artery wedge pressure decreased from previous levels, with considerable improvement in the pulmonary oedema, but all these patients developed very low right atrial pressures. Cardiac output and mean arterial pressure fell further, producing severe hypovolaemic shock. Attempts at volume repletion resulted in improvement in cardiac output and circulatory shock, but pulmonary artery wedge pressure and pulmonary oedema worsened.

Two echocardiographic studies have also shown severe systolic left ventricular dysfunction following envenomation. ${ }^{18} 22$ This has been attributed to catecholamine induced metabolic abnormalities in the myocardium, ${ }^{8}{ }^{10}$ increased myocardial oxygen requirements, ${ }^{20}$ myocardial ischaemia, ${ }^{9}$ and a direct effect of the toxin..$^{511} 12$ In the absence of previous data on peripheral vascular resistance, it was believed that, in addition to myocardial dysfunction, increased left ventricular afterload caused by systemic vasoconstriction also contributed to the left ventricular failure in patients with severe envenomation. ${ }^{131523}$ Our study clearly shows that systemic vascular resistance was not increased in these patients. Abroug et al also found that Tunisian patients with hypotension and pulmonary oedema had normal systemic vascular resistance. ${ }^{18}$ The reason for this reduction in systemic vascular resistance to normal in patients with severe envenomation is not clear. It may be the result of cholinergic stimulation, release of histamine, serotonin, and kinins, ${ }^{5}$ or a direct effect of the toxin on vascular smooth muscle ${ }^{35}$

Hypovolaemia may develop spontaneously in patients with envenomation as a result of the hot tropical environment, vomiting, excessive sweating and hypersalivation, ${ }^{5}$ or it may follow diuretic treatment. ${ }^{15}$ The rapid development of hypovolaemic shock in three of our patients with only mild dehydration suggests that these patients are extremely sensitive to small changes in blood volume.

A similar tendency to develop severe hypotension with mild dehydration has been described in phaeochromocytoma, as a result of decreased ventricular compliance and failure of vasoconstrictor reflexes. ${ }^{24-26}$ A previous study has also shown diastolic left ventricular dysfunction with decreased ventricular compliance in patients with scorpion envenomation. ${ }^{20}$ None of our patients had systemic vasoconstriction, which is the normal response to hypovolaemia.

Severe progressive cardiogenic shock was observed in one of our patients. The shock was refractory to inotropic drugs and probably represented the terminal phase of scorpionism, when right ventricular failure and peripheral vasodilatation combine with pre-existing left ventricular failure to cause profound irreversible circulatory failure.

EFFECT OF VASODILATOR TREATMENT WITH FLUID INFUSION

The use of inotropic drugs would seem an attractive option in patients with left ventricular dysfunction and pulmonary oedema. However, our past experience, as well as that of others, suggests that while inotropic drugs transiently improved circulatory failure they do not reduce mortality. ${ }^{15}$ This may be because inotropic catecholamines like adrenaline, dopamine, and dobutamine may aggravate myocardial damage produced by the already elevated circulating catecholamines. ${ }^{14}{ }^{15} \mathrm{We}$ therefore treated patients with captopril to reduce the left ventricular afterload, while giving fluid simultaneously to correct right ventricular preload or hypovolaemia. This resulted in a reduction in systemic vascular resistance and pulmonary artery wedge pressure, while cardiac output increased. The pulmonary oedema improved with only a minimal fall in mean arterial pressure. Over four to six hours, mean arterial pressure returned to normal. The treatment was continued for five to seven days in all patients.

Vasodilators have been used extensively in the treatment of pulmonary oedema following scorpion envenomation. Bawaskar and $\mathrm{Ba}-$ waskar have shown that mortality from scorpion envenomation in rural western India has been reduced to about $1 \%$ with the use of oral prazosin and intravenous nitroprusside infusion. ${ }^{1}{ }^{214}$ Angiotensin converting enzyme inhibitors have also been tried. ${ }^{15}$ However, as these drugs also inhibit kininase II leading to accumulation of bradykinin, their role in scorpion envenomation has been questioned. ${ }^{27}$ Our study shows that afterload reduction with the angiotensin converting enzyme inhibitor captopril improves the haemodynamic derangements and corrects pulmonary oedema.

\section{CONCLUSIONS}

Most investigators believe that pulmonary oedema in scorpion envenomation is cardiogenic, but others have suggested that it is caused by adult respiratory distress syndrome. Such observations have not been supported by measurement of pulmonary artery wedge pressure. All our patients with pulmonary oedema had increased pulmonary wedge pressures. Moreover, the course of pulmonary oedema closely paralleled changes in the wedge pressure, confirming its cardiogenic origin.

Although the numbers were small, our study has for the first time shown a haemodynamic basis for the various clinical syndromes seen in patients with scorpion envenomation. On the basis of the haemodynamic patterns observed, the following sequence of events could be postulated (fig 1). Patients with mild envenomation develop severe vasoconstriction and systemic hypertension. With severe envenomation, myocardial dysfunction with normal systemic vascular resistance occurs, resulting in 
acute left ventricular failure with normal right ventricular function. While initial hypertension progressing to left ventricular failure has been demonstrated in experimental animals injected with scorpion venom, this sequence has not been shown in humans. Depending on the circulating blood volume, left ventricular dysfunction may be manifested clinically as mild hypotension with severe pulmonary oedema in well hydrated patients, or severe hypotension with mild or no pulmonary oedema in the presence of dehydration. If untreated, loss of vascular tone and right ventricular failure may develop in addition to left ventricular failure, resulting in irreversible cardiogenic shock. Afterload reduction along with fluid infusion may correct many of the haemodynamic disturbances.

1 Bawaskar HS, Bawaskar PH. Scorpion envenoming and the cardiovascular system. Trop Doct 1997;27:6-9.

2 Bawaskar HS, Bawaskar PH. Treatment of envenomation by Mesobuthus tamulus (Indian red scorpion). Trans $R$ Soc Trop Med Hyg 1989;83:858-60.

3 Ramachandran LK, Agarwal OP, Achyutan KE, et al. Fractionation and biological activities of venoms of the Indian scorpions Buthus tamulus and Heterometrus bengalensis. Ind $\mathscr{f}$ Biochem Biophys 1986;23:355-8.

4 Russell FE. Toxic effects of animal toxins. In: Idaassen CD, Amdur MO, Doull J, eds. Toxicology - the basic science of poiAmdur MO, Doull J, eds. Toxicology - the basic science
sons, 3rd ed. New York: Macmillan, 1986:706-56.

5 Sofer S. Scorpion envenomation. Intensive Care Med Sofer S. Scorp

6 Moss J, Kazic T, Henry DP, et al. Scorpion venom-induced discharge of catecholamines accompanied by hypertension. Brain Res 1973;54:381-5.

7 Henriques MC, Gassinelli G, Diniz CR, et al. Effect of the venom of the scorpion Tityus serrulatus on adrenal gland catecholamines. Toxicon 1968;5:175-9.

8 Murthy KR, Vakil AE. Elevated plasma angiotensin levels in dogs by Indian red scorpion (Buthus tamulus) venom and its reversal by administration of insulin and tolazoline. Ind f Med Res 1988;88:376-9.

9 Margulis G, Sofer S, Zalstein E, et al. Abnormal coronary perfusion in experimental scorpion envenomation. Toxicon perfusion in experin

10 Murthy KR, Medh JD. Increase in serum free fatty acids, phospholipids and reduction in total cholesterol in acute
myocarditis produced by scorpion (Buthus tamulus) myocarditis produced by scorpion (Buthus tamulus)
venom injection in dogs. Ind Heart $\mathcal{F}$ 1986;38:369-71.
11 Wang R, Moreau P, Deschamps A, et al. Cardiovascular effects of Buthus martensii (Karsch) scorpion venom. Toxicon 1994;32:191-200

12 Almeida AP, Alpoim NC, Freire-Maia L. Effects of purified scorpion toxin (Tityus toxin) on the isolated guinea pig heart. Toxicon 1982;20:855-65.

13 Gueron M, Sofer S. Vasodilators and calcium channel blocking agents as treatment of cardiovascular manifestations of human scorpion envenomation. Toxicon 1990;28: $127-8$.

14 Bawaskar HS, Bawaskar PH. Prazosin for vasodilator treatment of acute pulmonary oedema due to scorpion sting. Ann Trop Med Parasitol 1987;87:719-23.

15 Karnad DR, Deo AM, Apte N, et al. Captopril for correcting diuretic-induced hypotension in pulmonary oedema after scorpion sting. BMF 1989;298:1430-1.

$16 \mathrm{El}$-Amin EO. Issues in management of scorpion sting in children. Toxicon 1992;30:111-15.

17 Campos JA, Silva OA, Lopez M, et al. Signs, symptoms and treatment of severe scorpion sting in children. Toxicon 1979;19(suppl 1):19.

18 Abroug E, Ayari M, Nouira S, et al. Assessment of left ventricular function in severe scorpion envenomation: combined hemodynamic and echo-Doppler study. Intensive Care Med 1995;21:629-35.

19 Gueron M, Ovsyhcher I. What is the treatment for the cardiovascular manifestations of scorpion envenomation? Toxicon 1987;25:121-4.

20 Gueron M, Adolph RJ, Grupp IL, et al. Hemodynamic and myocardial consequences of scorpion venom. Am $\mathrm{f}$ Cardiol 1980;45:979-86.

21 De Rezende NA, Dias MB, Campolina D, et al. Efficacy of antivenom therapy for neutralizing circulating venom antiantivenom in patients stung by Tityus serrulatus scorpions. Am f gens in patients stung by Tityus
Trop Med Hyg 1995;52:277-80.

22 Amaral CFS, Lopes JA, Magalhares RA, et al. Electrocardiographic, enzymatic and echocardiographic evidence of myocardial damage after Tityus serrulatus poisoning. $A m \mathcal{F}$ Cardiol 1991;67:655-7.

23 Bawsakar HS, Bawaskar PH. Management of cardiovascular manifestations of poisoning by the Indian red scorpion (Mesobuthus tamulus). Br Heart f 1992;68:478-80.

24 Pecoud A, Francioli P, Pradervand D, et al. Haemodynamics in pheochromocytoma. A report of 2 cases. Intensive Care Med 1979;5:143-6.

25 Stenstrom G, Kutti J. The blood volume in pheochromocytoma patients before and during treatment with phenoxybenzamine. Acta Med Scand 1985; 218:381-7.

26 Keiser $H$. Pheochromocytoma and related tumors. In: DeGroot LJ, Besser M, Burger HG, et al, eds. Endocrinology, 3rd ed. Philadelphia: W B Saunders, 1995:1853-77.

27 Ismail M, Fatani AJY, Dabees TT. Experimental treatment protocols for scorpion envenomation: a review of common therapies and an effect of kallikrein-kinin inhibitors. Toxicon 1992;30:1257-79. 